Article

\title{
Cellulose Isolation Methodology for NMR Analysis of Cellulose Ultrastructure
}

\author{
Marcus B. Foston, Chistopher A. Hubbell and Art J. Ragauskas *
}

School of Chemistry and Biochemistry, Institute of Paper Science and Technology, Georgia Institute of Technology, 500 10th St., Atlanta, GA 30332, USA;

E-Mails: marcus.foston@ipst.gatech.edu (M.B.F.); chis.hubbell@comcast.net (C.A.H.)

* Author to whom correspondence should be addressed; E-Mail: art.ragauskas@chemistry.gatech.edu; Tel.: +1-404-894-4578; Fax: +1-404-894-9701.

Received: 26 September 2011 / Accepted: 17 October 2011 / Published: 7 November 2011

\begin{abstract}
In order to obtain accurate information about the ultrastructure of cellulose from native biomass by ${ }^{13} \mathrm{C}$ cross polarization magic angle spinning (CP/MAS) NMR spectroscopy the cellulose component must be isolated due to overlapping resonances from both lignin and hemicellulose. Typically, cellulose isolation has been achieved via holocellulose pulping to remove lignin followed by an acid hydrolysis procedure to remove the hemicellulose components. Using ${ }^{13} \mathrm{C} \mathrm{CP} / \mathrm{MAS}$ NMR and non-linear line-fitting of the cellulose $\mathrm{C}_{4}$ region, it was observed that the standard acid hydrolysis procedure caused an apparent increase in crystallinity of $\sim 10 \%$ or less on the cellulose isolated from Populus holocellulose. We have examined the effect of the cellulose isolation method, particularly the acid treatment time for hemicellulose removal, on cellulose ultrastructural characteristics by studying these effects on cotton, microcrystalline cellulose (MCC) and holocellulose pulped Populus. ${ }^{13} \mathrm{C} \mathrm{CP} / \mathrm{MAS}$ NMR of MCC indicated that holocellulose pulping and acid hydrolysis has little effect on the crystalline ultrastructural components of cellulose. Although any chemical method to isolate cellulose from native biomass will invariably alter substrate characteristics, especially those related to regions accessible to solvents, we found those changes to be minimal and consistent in samples of typical crystallinity and lignin/hemicellulose content. Based on the rate of the hemicellulose removal, as determined by HPLC-carbohydrate analysis and magnitude of cellulose ultrastructural alteration, the most suitable cellulose isolation methodology utilizes a treatment of $2.5 \mathrm{M} \mathrm{HCl}$ at $100{ }^{\circ} \mathrm{C}$ for a standard residence time between 1.5 and $4 \mathrm{~h}$. However, for the most accurate crystallinity results this residence time should be determined empirically for a particular sample.
\end{abstract}


Keywords: acid hydrolysis; cellulose; crystallinity; isolation method; solid-state NMR; ${ }^{13} \mathrm{C}$ CP/MAS

\section{Introduction}

Bioethanol has been recognized as a potential source to replace current non-renewable resources via biochemical conversion from cellulose [1]. Lignocellulosics are the most abundant terrestrial organic materials, serving as a structural biopolymer in the cell walls of plants. The very properties that make lignocellulose so useful in nature, biomass recalcitrance, also makes it difficult and expensive deconstruct, especially biologically [2,3]. In order to meet global energy demands and produce sufficient biofuel volumes at lower cost, fundamental research has begun to focus on the chemistry, dynamics and mechanism of biomass recalcitrance and mitigation technologies [1,4].

Understanding the origins of biomass recalcitrance and the structural changes that occur during biosynthesis and deconstruction are vital for improving current processing and conversion methods for cellulosic biofuels. For example, efficient enzymatic hydrolysis of lignocellulose has been directly related to cellulase enzyme activity and the potential effect of substrate characteristics, such as crystallinity, degree of polymerization, specific surface area, lignin and hemicellulose distribution [5]. Researchers have employed plant genetic manipulation and thermochemical pretreatments to modify these substrate characteristics, reduce biomass recalcitrance and increase sugars yields from subsequent enzymatic deconstruction [6]. Unfortunately, these methods not only target plant cell wall characteristics of interest but also invariably change a variety of other substrate properties. In particular, hydrothermal pretreatments have been shown to increase enzymatic sugar yields, which have been subsequently correlated to changes in plant cell wall characteristics such as hemicellulose and lignin removal, increases in cellulose accessibility and biomass pore size and decreases in cellulose molecular weight [7-13]. Many of these same studies also indicate the crystallinity index of cellulose increases with hydrothermal pretreatment, however several studies have reported that a decrease in crystallinity may accelerate enzymatic hydrolysis $[9,14,15]$, demonstrating the rate of enzymatic hydrolysis is much faster with amorphous cellulose. However, the literature does not seem to present a consensus and along with recent work indicating accessibility maybe be one of the major first order rate determining factors [16], observed correlations between crystallinity and hydrolysis yield and rate does not indicate direction causation. This confusion can be contributed to the complex nature of the cell wall and again the fact that deconvolution of substrate properties to enzymatic hydrolysis requires capturing a comprehensive representation of the cell wall on multiple length scale, which few laboratories have the capabilities to accomplish. This suggests cellulose ultrastructure, though not as critical as once thought, is factor in enzymatic deconstruction of biomass which must be monitored if only to remove or confirm its minor affect on recalcitrance. Therefore, having techniques to accurately probe and track cellulose ultrastructure are critical to deconvoluting recalcitrant relevant plant cell wall characteristics and future improvements in the production of biofuels.

Infrared-red (IR) and Raman spectroscopy, along with X-ray diffraction (XRD) and electron diffraction/microscopic methodologies have been used to analyze cellulose ultrastructure; however no 
technique alone can fully characterize the ultrastructure of cellulose with respect to the proportions and exact nature of amorphous, crystalline and para-crystalline cellulose [17,18]. Another useful technique to analyze chemical and ultrastructural features of cellulose is nuclear magnetic resonance (NMR). NMR is sensitive to the magnetic non-equivalences in an environment of chemically equivalent nuclei. Consequently, ${ }^{13} \mathrm{C}$ cross polarization magic angle spinning (CP/MAS) NMR spectroscopy has been used to analyze the ultrastructure of cellulose for more than thee decades [15,18-40]. One of the first applications of ${ }^{13} \mathrm{C} \mathrm{CP} / \mathrm{MAS}$ NMR to investigate the ultrastructure of native cellulose was reported by Atalla and Vanderhart [18-20,26,27]. These studies employing cellulose from Acetobacter, Valonia, Kraft pulp and low-DP acid-hydrolyzed cellulose reported unique chemical shifts for the $\mathrm{C}_{4}$ and $\mathrm{C}_{6}$ carbon positions in the anhydroglucose unit dependent upon the cellulosic source. They proposed this was due to the rigid and highly hydrogen-bonded cellulose crystalline structure, in which the highly varied ultrastructure of cellulose, related to the cellulosic source, generated magnetic non-equivalences [18]. These non-equivalences were reflected in the spectra as variations in $\mathrm{C}_{4}$ and $\mathrm{C}_{6}$ carbon chemical shifts and related to the ultrastructure of cellulose.

The $C_{1}, C_{4}$ and $C_{6}$ signals of cellulose extend over chemical shift ranges of $\delta \sim 102-108,80-92$ and 57-67 ppm, respectively and display the best ${ }^{13} \mathrm{C}$ chemical shift dispersion of carbons in the anhydroglucose unit. Of those three regions, the $\mathrm{C}_{4}$ peak is most commonly used, and probes the cellulose amorphous domain which appears as a fairly broad signal from $\delta \sim 80-85 \mathrm{ppm}$. The $\mathrm{C}_{4}$ crystalline domain produces a sharper resonance from $\delta \sim 85-92 \mathrm{ppm}$ [18].

Typically, a two peak non-linear least-squared model or basic peak integration is used to determine the degree of crystallinity based upon the relative area of the amorphous and crystalline resonances in the $\mathrm{C}_{4}$ region. Park et al. [41] has reported a comprehensive comparison of degree of crystallinity as determined by solid state ${ }^{13} \mathrm{C}$ NMR utilizing either peak integration or a novel amorphous cellulose NMR spectral subtraction procedure to X-ray diffraction data analyzed by several different methods used to extract crystallinity information from diffraction patterns. This work not only described a new method for processing NMR spectra of cellulose to determine degree of crystallinity but also, and more importantly, demonstrated that NMR spectral fitting can accurately detect relative changes in crystallinity from different cellulose samples.

The NMR spectrum of cellulose, like many biological systems with complex chemical and structural moieties, contains broad and overlapping peaks. Via solvent exchange and ${ }^{13} \mathrm{C}$ spin-lattice relaxation experiments literature studies have identified several peak assignments within the spectrum of cellulose attributed to para-crystalline cellulose and amorphous accessible or inaccessible fibril surfaces. Resonances originating from para-crystalline cellulose and amorphous fibril surfaces displayed distinct $T_{1}$ relaxation rates on an order of magnitude shorter than the resonances attributed to cellulose $I_{\alpha}$ and cellulose $I_{\beta}[24]$. Also, accessible fibril surfaces demonstrated resonances displayed chemical shift changes after solvent exchange (not observed for inaccessible fibril surface related resonances) [24]. The exact nature and origins of resonances from para-crystalline cellulose and cellulose fibril surfaces has been an area of debate though the existence of these individual NMR-signals within the overlapping cellulose spectrum is well established. Work by Ding et al. [42] utilizing high-resolution atomic force microscopy on the surface of cellulose microfibrils suggested that the elementary cellulose fibril is composed of 36 glucan chains, where the most inner core six chains are true crystalline chains. Those core chains are surrounded by 12 para- or sub-crystalline 
chains, which are then surrounded by 18 more sub-crystalline or non-crystalline chains. NMR methodologies not only estimate relative amounts of cellulose crystal allomorphs but also deconvolute contributions of para-crystalline cellulose, accessible and inaccessible fibril surfaces, which has been refined to include the non-linear line-fitting of six or seven resonances of adjustable shape, width, chemical shift, and relative intensity.

The determination of cellulose crystallinity and spectral fitting of NMR data to estimate the relative amounts of the various ultrastructural components of cellulose is fairly straightforward on pure samples of cellulose [24,31]. In 1994, Lennholm et al. [31] developed a novel method utilizing a partial least-squares (PLS) model, a precursor to the analysis described above, to estimate the relative amounts of cellulose crystallinity index involving cellulose $I_{\alpha}$ and cellulose $I_{\beta}$-content on variety of calibration samples such as cellulose from Valonia ventricosa, bacterial cellulose from Acetobacter xylinum, cotton linters, etc. However, wood pulp and other biomass samples contain other overlapping NMR-signals from hemicellulose and lignin side-chains that can contribute to the signal intensity of resonances of interest. Earlier work by Newnan et al. [39] used spectral editing NMR sequences, specifically a $T_{1 \rho}$ spin-lock filter prior to the contact pulse in the CP sequence to probe crystallinity in wood pulp samples $[32,40]$. There are several other spectral editing techniques based on other $\mathrm{T}_{2}$ and $\mathrm{T}_{1}$ relaxation phenomena that have been reported [32,39,43]. In general, spectral editing techniques work by allowing most of the NMR-signal of hemicellulose and lignin side-chains to decay or relax while retaining the signal from cellulose due to differences in relaxation rates. Though this technique is particularly useful in qualitative analyses of wood samples, the fact that crystalline and amorphous cellulose have different relaxation rates makes extracting quantitative information difficult.

In an effort to further study the non-crystalline forms within biomass containing cellulose I by ${ }^{13} \mathrm{C}$ CP/MAS NMR Larsson et al. [22] prepared bleached birch kraft pulp with a method originally cited to prepare hydrocellulose [44] by refluxing in $2.5 \mathrm{M} \mathrm{HCl}$ for various residence times. In doing so, they proposed four hours of hydrolysis time was the optimal compromise between removing most of the hemicelluloses with minimal cellulose degradation. Following this study, the standard sample preparation of cellulose from wood for ultrastructural analysis by ${ }^{13} \mathrm{C} C \mathrm{CP} / \mathrm{MAS} \mathrm{NMR}$ involved removing the lignin component by holocellulose pulping followed by an acid hydrolysis to remove the hemicellulose component. Alkaline extraction of hemicellulose is also very effective at the removal of hemicellulose and may cause less severe degradation of residual cellulose, however Iverson and other researchers have avoided this method due to cellulose mercerization which may convert cellulose I to the cellulose II $[24,45,46]$.

There are other complementary methods, primarily X-ray diffraction and IR spectroscopy, which have been employed to determine cellulose crystallinity in wood fibers [3,47]. However, crystallinity data from these different analytical techniques has been shown to demonstrate dissimilarity in results on the same sample, most likely due to varying principles of measurement. In the aforementioned study by Park et al. [41], eight different cellulose preparations produced significantly different crystallinity values depending on the method used. Nevertheless, for each technique tested the resulting crystallinity index of the various cellulose preparations ranked similarly and the relative changes seemed consistent. Moreover, with both techniques, obscuring signals from lignin and hemicelluloses can complicate accurate analysis of the cellulose ultrastructure. This therefore 
makes cellulose isolation and determining the effect of cellulose isolation on the ultrastructure even more relevant.

In this study, we examine in greater detail ${ }^{13} \mathrm{C} C \mathrm{C} / \mathrm{MAS}$ NMR ultrastructural analysis of cellulose in conjunction with HPLC carbohydrate analysis to assess residual hemicellulose distributions and gel permeation chomatography (GPC) to probe cellulose degradation. All these studies were performed to determine the effect of cellulose isolation methodologies on cellulose and its ultrastructure. Holocellulose pulped Populus, cotton and microcrystalline cellulose (MCC) samples were refluxed in $2.5 \mathrm{M} \mathrm{HCl}$ for various residence times in an attempt to further define the changes occurring in the ultrastructure of cellulose during these chemical treatments used to isolate cellulose and how that could alter our interpretation of the resulting spectral data.

\section{Results and Discussion}

To comprehensively study the effect of cellulose isolation method on the ultrastructure of cellulose, we chose to first evaluate the efficiency of hemicellulose removal via HPLC carbohydrate analysis of a time-series of acid hydrolysis reactions on holocellulose pulped Populus. Once a rate on hemicellulose extraction is established, the magnitude of changes in the ultrastructure of cellulose by ${ }^{13} \mathrm{C} \mathrm{CP} / \mathrm{MAS}$ NMR and cellulose degradation rates as acquired by GPC were determined. This was done on a variety of substrates (cotton, microcrystalline cellulose and holocellulose pulped Populus) and used to model native cellulose within a cell wall, evaluating changes in the ultrastructure of cellulose during hemicellulose extraction.

\subsection{Hemicellulose Removal}

To effectively analyze the ultrastructure of cellulose using ${ }^{13} \mathrm{C} \mathrm{CP} / \mathrm{MAS}$ NMR the majority of lignin and hemicellulose, which contain signals in the same spectral region as the $\mathrm{C}_{4}$ resonance, must be removed. The removal of hemicellulose relies on an acid hydrolysis procedure that was reported by Larsson et al. $[22,25,44]$. In order to develop a more complete description of the changes occurring to the ultrastructure of cellulose a time-resolved study of the hemicellulose removal procedure from holocellulose pulped Populus included refluxing in $2.5 \mathrm{M} \mathrm{HCl}$ for varying times up to $300 \mathrm{~min}$. HPLC-based monosaccharide anionic exchange chomatography of the pulp was used to quantitatively analyze the changes in the composition of the residual cell wall carbohydrates. The hemicellulose content, characterized by the xylose, mannose, arabinose, and galactose contents are combined, whereas the cellulose content is represented by the glucose content. Figure 1a summarizes the changes in carbohydrate distribution at various points during the acid hydrolysis. The first data points in Figure 1a indicate that holocellulose pulping removes almost no hemicellulose sugars and that removal of hemicellulose by acid hydrolysis to only be significant after $30 \mathrm{~min}$. The hemicellulose content seemingly plateaus at $1-2 \%$ after 90 min of reflux under the reaction conditions employed. 
Figure 1. (a) Carbohydrate distribution and (b) reciprocal of the hemicellulose (Hemi) content for a Populus standard after holocellulose pulping and hemicellulose removal by acid hydrolysis in $2.5 \mathrm{M} \mathrm{HCl}$ at various residence times.

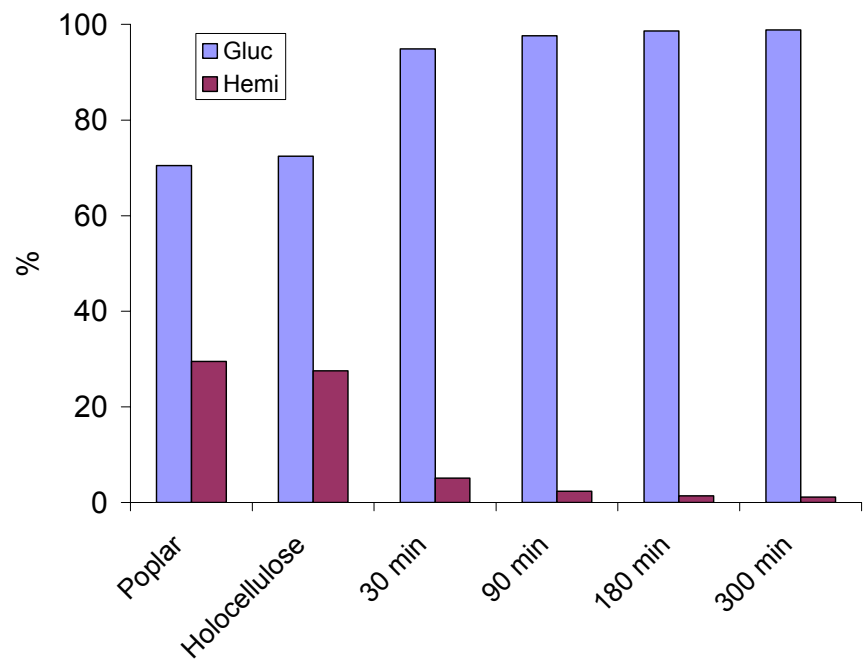

(a)

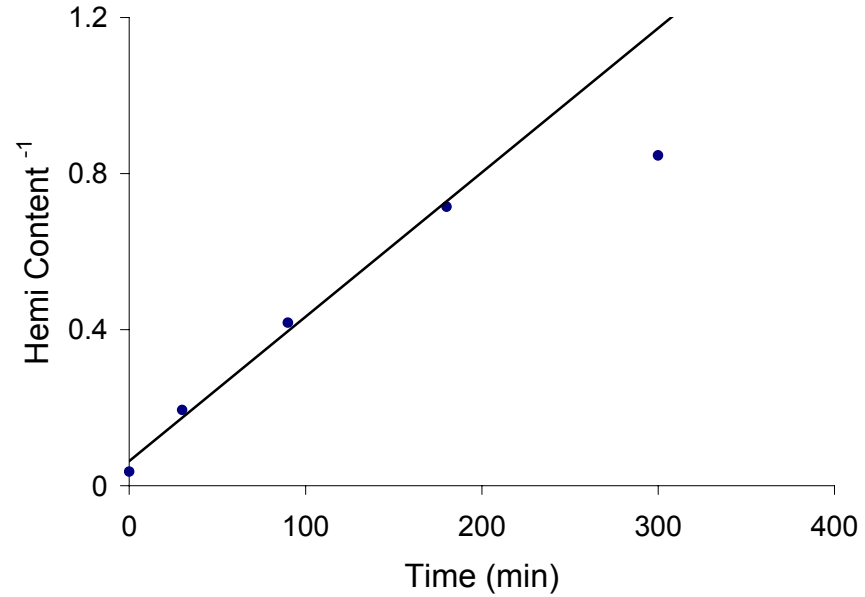

(b)

Plotting the reciprocal of the residual hemicellulose content versus time during acid hydrolysis, as seen in Figure 1b, provides a linear relationship suggesting a second order kinetic for the first $\sim 3 \mathrm{~h}$. Based upon these results, the reaction half-life of hemicellulose removal is $\sim 13.5 \mathrm{~min}$, though this value is not completely accurate as it seems the rate of hemicellulose removal slows with reaction time, deviating from a single second order rate constant near the end of the hydrolysis treatment. Utilizing this rate, alterations within the cellulose ultrastructure and cellulose degradation can be quantitatively compared to hemicellulose removal in a continuous fashion.

\subsection{Changes in Ultrastructure and Crystallinity}

In an effort to better understand the ultrastructural changes occurring within cellulose during cellulose isolation our initial studies focused on microcrystalline cellulose (MCC). MCC is prepared by exposing a bleached delignified cellulosic source to a mineral acid solution which removes some of the cellulose amorphous regions and leaves an enriched crystalline microfibril. Its ubiquitous use as model cellulose substrate and the highly crystalline nature of MCC substrate makes it an attractive substrate to analyze potential alterations to the relative proportions of crystalline allomorphs during holocellulose pulping and the acidic hemicellulose extraction treatment.

In order to probe changes occurring in the cellulose crystalline ultrastructure, the relative intensity of the ultrastructural components within cellulose microfibrils and how those relative intensities change with various treatments were evaluated by ${ }^{13} \mathrm{C} \mathrm{CP} / \mathrm{MAS}$ NMR as seen in Figure 2. Crystallinity was determined via 2-peak integration of the $C_{4}$ crystalline carbon region ( $\delta$ 85-92 ppm) over the integral of the entire $\mathrm{C}_{4}$ region $(\delta 80-92 \mathrm{ppm})$. Also, ultrastructural components in the crystalline regions of cellulose were derived by line-fitting one Gaussian and thee Lorentzian line-shapes to the $\mathrm{C}_{4}$ crystalline carbon signals from $\delta$ 85-92 ppm, which are attributed to domains of cellulose $\mathrm{I}_{\alpha+\beta}, \mathrm{I}_{\alpha}, \mathrm{I}_{\beta}$ and para-crystalline cellulose, a procedure described in the literature [24,31] (non-crystalline contributions to crystalline carbon signals were accounted for by the simultaneous mathematical treatment of the $\delta$ 
80-95 ppm region). The non-linear line-fit of the untreated samples are shown in Figure 3, with the corresponding line parameters in Supplementary-Tables S1 and S2. Lateral fibril dimensions (LFD) can be estimated using the relative intensity of peaks attributed to amorphous cellulose, considered in a square cross-sectional cellulose microfibril model for NMR analysis by Huex et al. [48] as total fibril surfaces $[43,49]$. Based on those intensities and the microfibril model comprised of chains having a width of $0.55 \mathrm{~nm}$, the LFD were also estimated as reference, elucidating the size of the fibril in which the various phases of cellulose $\mathrm{I}_{\alpha}, \mathrm{I}_{\beta}$ and para-crystalline cellulose exist (see Table 1).

Figure 2. ${ }^{13} \mathrm{C} \mathrm{CP} / \mathrm{MAS}$ spectra of microcrystalline cellulose (MCC) and cellulose from cotton swaps after various treatments used to isolate cellulose from whole biomass samples. The \pm value represents thee standard deviation of typical variation associated with the measurement. Un = untreated, Hemi = hemicellulose removal by acid hydrolysis, $\mathrm{H}=$ holocellulose pulped, $\mathrm{HH}=$ holocellulose pulped and hemicellulose removal by acid hydrolysis.

MCC

Cotton

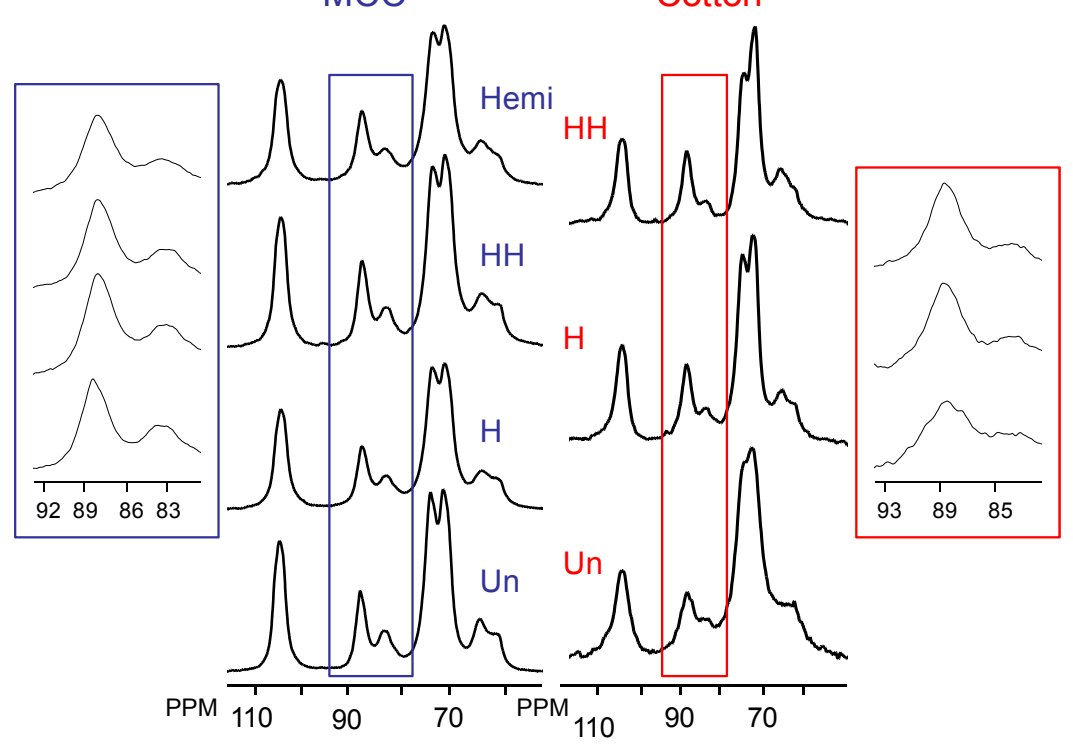

Figure 3. Non-linear line-fits of the ${ }^{13} \mathrm{C} \mathrm{CP} / \mathrm{MAS}$ spectra of untreated microcrystalline cellulose (MCC) and cellulose from cotton swaps.

MCC

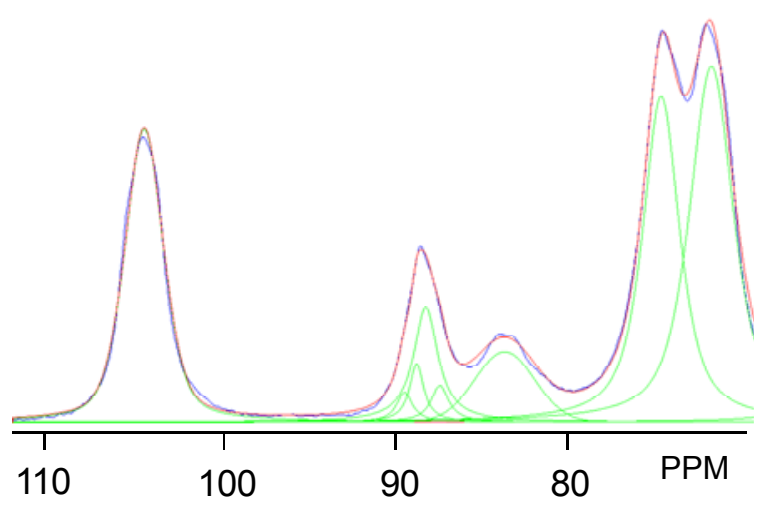

Cotton

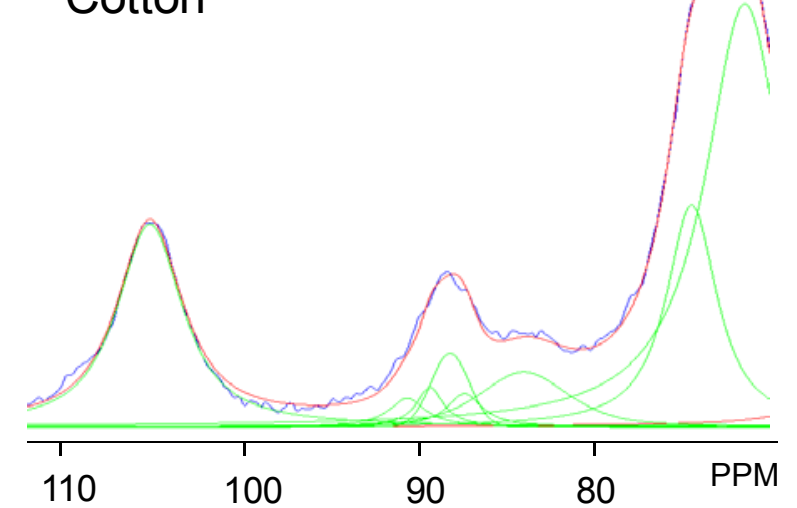


Table 1. Crystalline ultrastructural results for line-fitting of ${ }^{13} \mathrm{C} \mathrm{CP} / \mathrm{MAS}$ spectra of microcrystalline cellulose (MCC) and cellulose from cotton swaps after various treatments used to isolate cellulose from whole biomass samples. The \pm value represents thee standard deviation of typical variation associated with the measurement. Un $=$ untreated, Hemi $=$ hemicellulose removal by acid hydrolysis, $\mathrm{H}=$ holocellulose pulped, $\mathrm{HH}=$ holocellulose pulped and hemicellulose removal by acid hydrolysis. The plus-minus values represent thee standard deviations of typical variation associated with the measurement.

\begin{tabular}{ccccccc}
\hline Sample & $\begin{array}{c}\mathbf{I}_{\boldsymbol{\alpha}}(\boldsymbol{\%}) \\
\mathbf{\pm 3}\end{array}$ & $\begin{array}{c}\mathbf{I}_{\boldsymbol{\alpha}+\boldsymbol{\beta}}(\mathbf{\%}) \\
\mathbf{\pm 4}\end{array}$ & $\begin{array}{c}\text { Para }(\boldsymbol{\%}) \\
\mathbf{\pm 6}\end{array}$ & $\begin{array}{c}\mathbf{I}_{\boldsymbol{\beta}}(\boldsymbol{\%}) \\
\mathbf{\pm 6}\end{array}$ & $\begin{array}{c}\mathbf{C r I} \mathbf{( \% )} \\
\mathbf{\pm 2}\end{array}$ & $\begin{array}{c}\text { LFD (nm) } \\
\mathbf{\pm 0 . 5}\end{array}$ \\
\hline Un MCC & 8 & 11 & 35 & 8 & 62 & 5.2 \\
H MCC & 7 & 8 & 35 & 10 & 60 & 4.9 \\
HH MCC & 6 & 10 & 42 & 11 & 68 & 6.3 \\
Hemi MCC & 7 & 11 & 40 & 19 & 66 & 5.9 \\
Un cotton & 9 & 9 & 35 & 9 & 62 & 5.2 \\
H cotton & 6 & 13 & 40 & 6 & 66 & 5.9 \\
HH cotton & 6 & 14 & 49 & 6 & 79 & 9.9 \\
\hline
\end{tabular}

The data in Table 1 represents the results of this fitting procedure on ${ }^{13} \mathrm{C}$ spectra of MCC which has undergone the hemicellulose extraction procedure along with other procedures used to isolated cellulose from native biomass. This data suggest little or no change occurs with respect to the crystallinity with holocellulose pulping. More importantly, there seems to only be a slight increase in crystallinity upon treatment under the conditions used for acid hydrolysis of hemicellulose. However, this may be due, in part, to the inherently high proportion of crystalline cellulose which results from the preparation of MCC. Therefore, in an effort to observe the ultrastructural changes occurring within cellulose during cellulose isolation on a substrate with an ultrastructure more comparable to native cellulose a cotton sample was studied using the same protocols used on MCC. As summarized in Table 1, the relative proportions of the cellulose $\mathrm{I}_{\alpha}, \mathrm{I}_{\beta}$ and para-crystalline cellulose were found not to undergo any significant changes when holocellulose pulped.

The crystallinity and relative proportion of para-crystalline cellulose in cellulose from cotton does however appreciably increase upon acid hydrolysis. This change in ultrastructural behavior is indicative of the increased amorphous content in the cotton, suggesting spatially localized hydrolysis preferably degrades amorphous cellulose, increasing the crystallinity. This additional crystallinity is perceived as sub-crystalline cellulose units. This later point will be further discussed with respect to cellulose degradation and is a key point in the overall conclusions of this work.

The plot in Figure 4 shows the change in crystallinity with increasing residence time in the hemicellulose removal procedure on holocellulose Populus. The $\mathrm{C}_{4}$ signals from the cellulose and hemicellulose peaks overlap even in partially acid hydrolyzed samples which can skew the resulting degree of crystallinity determination for cellulose. In an effort to overcome the presence of the hemicellulose resonances, both traditional ${ }^{13} \mathrm{C} \mathrm{CP}$ experiments and $\mathrm{CP}$ experiments with a $6.5 \mathrm{~ms}{ }^{1} \mathrm{H}$ spin-lock filter (a published and validated procedure to estimate the degree of crystallinity in pulps) were conducted and reported in Figure 4. Hemicellulose ${ }^{13} \mathrm{C}$ NMR signals arise at $\delta 83.5$ and 
$81.7 \mathrm{ppm}$ [50]. In addition, the hemicellulose acetyl groups of Populus with resonances at $\delta \sim 174$ and $21.5 \mathrm{ppm}$ [51] were readily observed. Experimental ${ }^{1} \mathrm{H} \mathrm{T}_{1 \rho}$ values for crystalline and non-crystalline cellulose $\mathrm{C}_{4}$ protons in bleached Kraft pulps determined by Newman et al. [39], which were similar to values measured in this study, were $\sim 13.6$ and $12.7 \mathrm{~ms}$ [39], respectively. Whereas the acetate group displayed a much lower ${ }^{1} \mathrm{H} \mathrm{T}_{1 \rho}$ value of $\sim 5.0 \mathrm{~ms}$, [39] this could in part be due to the additional rotational motion of the $O$-methyl bond. However, Newman et al. [39] concluded that the acetate groups are so intimately distributed in the hemicellulose that nuclear spin diffusion results in an average $T_{1 \rho}$ value. Therefore, the spin-lock filter time was determined by the characteristic ${ }^{1} \mathrm{H} \mathrm{T}_{1 \rho}$ time of the acetate group at $\delta 21.5 \mathrm{ppm}$ and cellulose $\mathrm{C}_{4}$ resonance, allowing a sufficient portion of the hemicellulose magnetization to attenuate during spin-locking before cross-polarization [39]. Both curves display very similar rates of increase with respect to crystallinity and seem to suggest an increase in crystallinity of $\sim 10 \%$ or less on cellulose isolated from Populus holocellulose after refluxing for $4 \mathrm{~h}$ in $2.5 \mathrm{M} \mathrm{HCl}$. Untreated Populus standard after holocellulose pulping showed a \% crystallinity of 53 and 40 for the normal and spin-lock filtered ${ }^{13} \mathrm{C} \mathrm{CP}$ sequence, ultimately increasing to 64 and $61 \%$ after 5 hours of hemicellulose removal by acid hydrolysis in $2.5 \mathrm{M} \mathrm{HCl}$.

Figure 4. Degree of crystallinity of cellulose from a Populus standard after holocellulose pulping and hemicellulose removal by acid hydrolysis in $2.5 \mathrm{M} \mathrm{HCl}$ at various residence times as measured by a ${ }^{13} \mathrm{C} \mathrm{CP}$ sequence $(\bullet)$ and ${ }^{13} \mathrm{C} \mathrm{CP}$ sequence with $6.5 \mathrm{~ms}{ }^{1} \mathrm{H}$ spin-lock filter ( $\mathbf{\square})$. The dotted line represents the normal $4 \mathrm{~h}$ reaction time for hemicellulose removal typically employed. The error bars represent thee standard deviations of typical variation associated with the measurement.

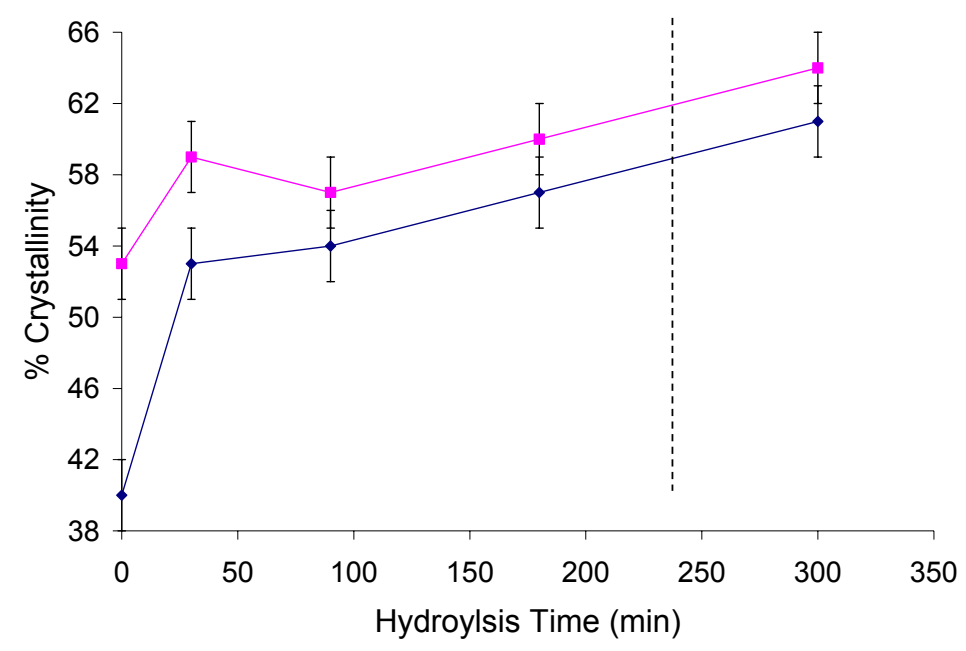

\subsection{Cellulose Degradation}

To assess possible degradation and changes in the cellulose ultrastructure due to acid hydrolysis during the aforementioned isolation procedures, a sample of MCC and cotton was holocellulose pulped and refluxed in $2.5 \mathrm{M} \mathrm{HCl}$ and the changes in number- and weight-average DP were determined and plotted in Figure 5. There are several methods, including vapor pressure osmometry, reducing end concentration, electron microscopy, light scattering, sedimentation equilibrium, X-ray small angle scattering and intrinsic viscosity, which can be used to determine the relative molecular weight of 
cellulose [16]. However a commonly utilized technique generates cellulose tricarbanilate with phenyl isocyanate to facilitate dissolution in tetrahydrofuran (THF) and elution in a gel permeation chomatographic (GPC) system. This method requires dissolution by derivatization, specialized chomatography and external standards; however, unlike other direct methods such as the phenol-sulfuric acid method [52] is the only technique which provides a true molecular weight distribution.

As expected, holocellulose pulping conditions had little effect on the MCC molecular weight (see Figure 5). However, significant reductions in DP were observed for cellulose obtained from cotton when subjected to holocellulose pulping. These observations are similar and supported by recent studies by Hubbell et al. [53] which showed MCC and cotton linters with 30\% lignin manually incorporated into the samples had only a 1 and $12 \%$ reduction in DP, respectively. However, in comparison a 5 and 35\% reduction in DP for the 'lignin-free' samples were reported upon acid-chlorite holocellulose pulping. In particular, the results on the cotton linters seem to suggest that even though amorphous components of cellulose may display a significant rate of hydrolysis during holocellulose pulping condition when the cellulose is encased in a hemicellulose and lignin matrix the degradation is nominal. When analyzing the effect of holocellulose pulping on cotton in this study, a significant reduction in DP was detected after hemicellulose removal on the substrate, which again would be mitigated by the presence of lignin and hemicellulose in an intact cell wall.

Figure 5. Degree of polymerization of microcrystalline cellulose and cellulose from cotton swaps after various treatments used to isolate cellulose from whole biomass samples. The error bars represent the standard deviations. Hemi $=$ hemicellulose removal by acid hydrolysis, $\mathrm{H}=$ holocellulose pulped, $\mathrm{HH}=$ holocellulose pulped and hemicellulose removal by acid hydrolysis.

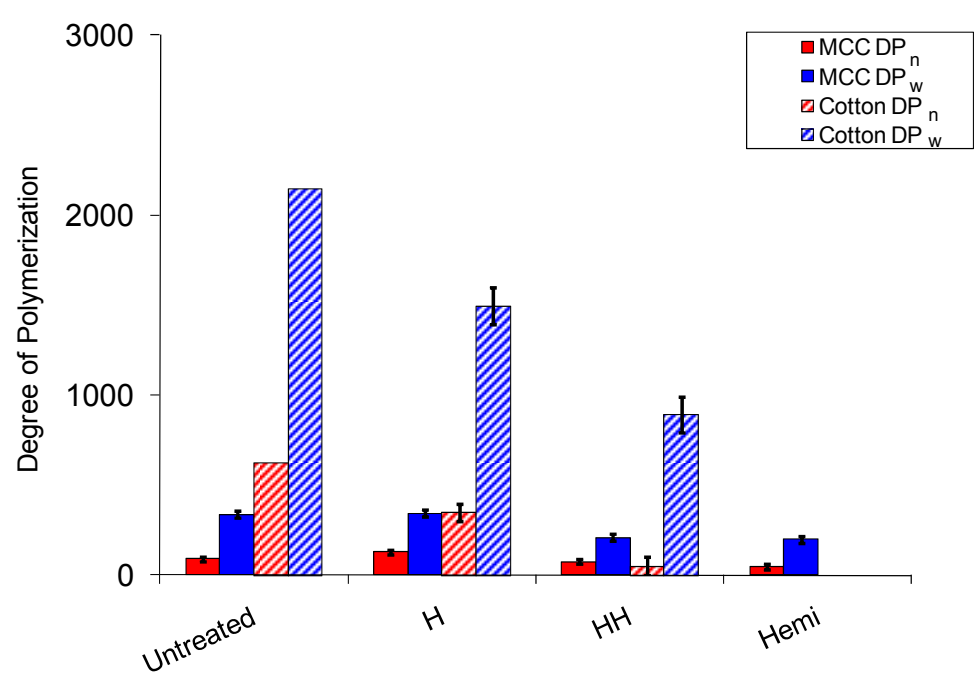

A key consideration in determining the proper conditions to remove hemicellulose from biomass is the efficiency of hemicellulose removal versus the degradation of cellulose. GPC characterization of $\alpha$-cellulose [54] isolated from Populus was studied in a similar time-resolved fashion to establish the rate at which cellulose is degraded by the acid hydrolysis. Figure 6 shows a very typical scheme used to represent cellulose degradation, a plot related to number of chain breaks with respect to the initial number-average DP of cellulose as a function of reaction time, where the dotted line represents the $4 \mathrm{~h}$ 
typically associated with the hemicellulose extraction procedure. The results in Figure 4 are similar to a more detailed study by Stephens et al. [55] conducted on the hydrolysis of the amorphous cellulose in cotton-based paper. They determined that degradation by acid hydrolysis can be considered as having thee major stages. The initial stage was described as rapid hydrolytic attack on the more solvent accessible amorphous chain segments, while the latter stages display a much slower hydrolytic attack breaking amorphous chain segments on and/or at crystal surfaces. More importantly, the overall degradation process was characterized as exponential having a first-order kinetic. The increase in the number of cellulose chain breaks seen in Figure 4 occurs as an exponential function, suggesting a first order kinetic for the hydrolysis of cellulose during the hemicellulose acid hydrolysis procedure. The average reaction half-life of cellulose degradation during acid hydrolysis in both amorphous and crystalline regions ( $\sim 79 \mathrm{~min})$ is about 6 times that of hemicellulose removal. Correspondingly, the only large increases in crystallinity occurred in samples which were small molecule extracted, holocellulose pulped and acid hydrolyzed to remove hemicellulose. These same samples consistently were also associated with cellulose degradation equaling \# of chain breaks $>2$.

Figure 6. Number of chain breaks of cellulose from a Populus standard after holocellulose pulping and hemicellulose removal by acid hydrolysis in $2.5 \mathrm{M} \mathrm{HCl}$ at various residence times $\left(\#\right.$ Chain Breaks $\left.=\left(\mathrm{DP}_{\mathrm{n}}{ }^{\mathrm{o}} / \mathrm{DP}_{\mathrm{n}}\right)-1\right)$. The dotted line represents the normal $4 \mathrm{~h}$ reaction time for hemicellulose removal typically employed and the solid line represents an exponential least-squared fit ( $\#$ of chain breaks $=\mathrm{B}+\mathrm{A}^{*} \exp (\mathrm{time} / \tau) ; \mathrm{B}=2.1 ; \mathrm{A}=-2.0$; $\tau=114.9 \mathrm{~min})$.

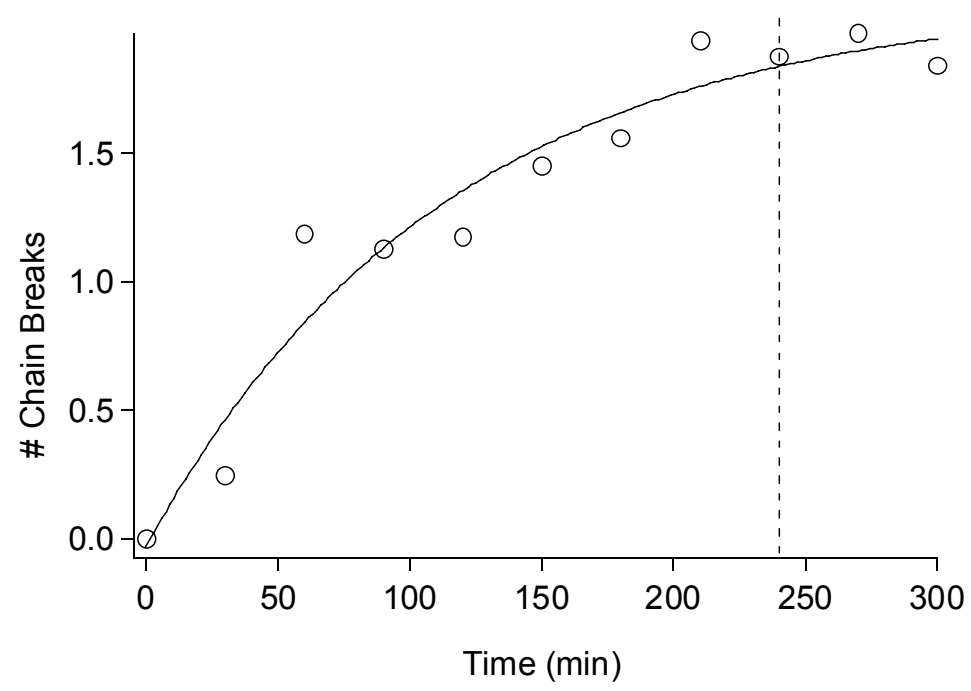

Alternative methods of ultrasound- or organic solvent-assisted acid/base extraction of hemicellulose were also attempted in our lab and did not appreciably improve the hemicellulose extraction methodology. The results of this work clearly indicate there is no method to isolate cellulose from lignocellulosic biomass which can leave the cellulose in an unchanged, native state. The use of enzymes to remove both lignin and hemicellulose can be found in a few literature examples, yielding cellulose in almost quantitative yield however the impurities in the cellulose (residual proteins) can be substantial [56]. These impurities can be difficult to remove and make subsequent analytical techniques measuring chemical- or ultrastructure difficult. Furthermore, only about a third of the xylan 
present in birch pulp was found to be accessible to digestion by xylanases or extraction with 5 wt $\%$ potassium hydroxide [50], which was considered dilute enough to not cause mercerization.

\section{Experimental Section}

\subsection{Substrates}

Populus (Populus trichocarpa $x$ deltoides) samples were harvested between 2007-2008 by National Renewable Energy Laboratory (NREL) at area 0800 at Oak Ridge National Laboratory, TN, USA. Microcrystalline cellulose and cotton were purchased from Sigma-Aldrich (St. Louis, MO, USA) and used as received. The biomass was size-reduced in a Wiley mill to a maximum particle size of $0.841 \mathrm{~mm}$, and then fines less than $0.177 \mathrm{~mm}$ were removed with a sieve screen. Extractives were subsequently removed by placing the biomass into an extraction thimble in a Soxhlet extraction apparatus. The extraction flask was filled with 1:2 ethanol/toluene mixture $(\sim 150 \mathrm{~mL})$ and then refluxed at a rate which cycled the biomass for at least 24 extractions over a $4 \mathrm{~h}$ period. The extracted samples were then air-dried prior to further study.

\subsection{Carbohydrates and Klason Lignin Analysis}

Samples for carbohydrate constituents and acid-insoluble residue (Klason lignin) analysis milled Populus were prepared using a two-stage acid hydrolysis protocol based on TAPPI methods T-222 om-88 with a slight modification. The first stage utilizes a severe $\mathrm{pH}$ and a low reaction temperature (72 vol. $\% \mathrm{H}_{2} \mathrm{SO}_{4}$ at $30{ }^{\circ} \mathrm{C}$ for $1 \mathrm{~h}$ ). The second stage is performed at much lower acid concentration and higher temperature ( 3 vol. $\% \mathrm{H}_{2} \mathrm{SO}_{4}$ at $121{ }^{\circ} \mathrm{C}$ for $1 \mathrm{~h}$ ) in an autoclave. The resulting solution was cooled to room temperature and filtered using G8 glass fiber filter (Fisher Scientific, Hanover Park, IL, USA). The remaining residue which is considered as Klason lignin was oven-dried and weighed to obtain the Klason lignin content. The filtered solution was analyzed for carbohydrate constituents of the hydrolyzed Populus samples determined by high-performance anion-exchange chomatography with pulsed amperometric detection (HPAEC-PAD) using Dionex ICS-3000 (Dionex Corp., Sunnyvale, CA, USA) [57].

\subsection{Holocellulose Pulping}

Isolated cellulose was generated by first isolating holocellulose from extracted milled biomass pulp. Holocellulose was isolated from extracted samples by exposure to $\mathrm{NaClO}_{2}(1.30 \mathrm{~g} / 1.00 \mathrm{~g}$ lignocellulosic dry solids) in acetic acid $(375 \mathrm{~mL}$ of $0.14 \mathrm{M})$ at $70{ }^{\circ} \mathrm{C}$ for $2 \mathrm{~h}$. The samples were then collected by filtration and rinsed with an excess of DI filtered water. This was repeated at least once to ensure removal of the majority of the lignin component and then washed with DI water to yield holocellulose pulped samples. Holocellulose samples were shown to contain $\sim 3.7 \%$ Klason lignin after pulping twice.

\subsection{Cellulose Isolation for GPC Analysis}

Isolated cellulose was prepared from the holocellulose sample (1.00 g) by extraction with a $17.5 \%$ $\mathrm{NaOH}$ solution $(50 \mathrm{~mL})$ at $25^{\circ} \mathrm{C}$ for $30 \mathrm{~min} .50 \mathrm{~mL}$ of DI filtered water was then added to the $\mathrm{NaOH}$ 
solution. The extraction was continued with the $8.75 \% \mathrm{NaOH}$ solution $(43 \mathrm{~mL})$ at $25{ }^{\circ} \mathrm{C}$ for an additional $30 \mathrm{~min}$. Cellulose isolated in this manner is commonly referred to as $\alpha$-cellulose. The isolated $\alpha$-cellulose samples were then collected by filtration and rinsed with $50 \mathrm{~mL}$ of $1 \%$ acetic acid, an excess of DI filtered water, and air dried.

\subsection{Gel Permeation Chomatography (GPC) Analysis of Cellulose}

The number-average molecular weight $\left(\mathrm{M}_{\mathrm{n}}\right)$ and weight-average molecular weight $\left(\mathrm{M}_{\mathrm{w}}\right)$ were determined by GPC after tricarbanilation of cellulose [6,54]. Lignin-free cellulose (15 mg) from each sample was placed in separate test tubes equipped with micro stir-bars and dried overnight under vacuum at $40{ }^{\circ} \mathrm{C}$. The test tubes were then capped with rubber septa. Anhydrous pyridine $(4.00 \mathrm{~mL})$ and phenyl isocyanate $(0.50 \mathrm{~mL})$ were added sequentially via syringe. The test tubes were placed in an oil bath at $70{ }^{\circ} \mathrm{C}$ and allowed to stir for $48 \mathrm{~h}$. Methanol $(1.00 \mathrm{~mL})$ was added to quench any remaining phenyl isocyanate. The contents of each test tube were then added dropwise to a 7:3 methanol/water mixture $(43 \mathrm{~mL})$ to promote precipitation of the derivatized cellulose. The solids were collected by filtration and then washed with methanol/water $(1 \times 50 \mathrm{~mL})$ followed by water $(2 \times 50 \mathrm{~mL})$. The derivatized cellulose was then dried overnight under vacuum at $40{ }^{\circ} \mathrm{C}$. Prior to GPC analysis the derivatized cellulose was dissolved in THF $(1 \mathrm{mg} / \mathrm{mL})$, filtered though a $0.44 \mu \mathrm{m}$ filter and placed in a $2 \mathrm{~mL}$ auto-sampler vial.

The molecular weight distributions of the cellulose tricarbanilate samples were then analyzed on an Agilent GPC SECurity 1200 system equipped with four Waters Styragel columns (H1, H2, H4, H6), Agilent refractive index (RI) detector and Agilent UV detector (270 nm) using THF as the mobile phase $(1.0 \mathrm{~mL} / \mathrm{min})$ with injection volumes of $20 \mu \mathrm{L}$. A calibration curve was constructed based on eight narrow polystyrene standards ranging in molecular weight from $1.5 \times 10^{3}$ to $3.6 \times 10^{6} \mathrm{~g} / \mathrm{mol}$. Data collection and processing were performed using Polymer Standards Service WinGPC Unity software (Build 6807). Molecular weights $\left(\mathrm{M}_{\mathrm{n}}\right.$ and $\left.\mathrm{M}_{\mathrm{w}}\right)$ were calculated by the software relative to the polystyrene calibration curve. Number-average degree of polymerization $\left(\mathrm{DP}_{\mathrm{n}}\right)$ and weight-average degree of polymerization ( $\left(\mathrm{P}_{\mathrm{w}}\right.$ ) were obtained by dividing $\mathrm{M}_{\mathrm{n}}$ and $\mathrm{M}_{\mathrm{w}}$ by $519 \mathrm{~g} / \mathrm{mol}$, the molecular weight of the tricarbanilated cellulose repeat unit. Polydispersity index (PDI) was calculated by dividing $\mathrm{M}_{\mathrm{w}}$ by $\mathrm{M}_{\mathrm{n}}$.

\subsection{Cellulose Isolation for NMR Analysis}

Isolated cellulose was prepared from the holocellulose sample $(1.00 \mathrm{~g})$ by hydrolysis for $4 \mathrm{~h}$ in $\mathrm{HCl}$ $(43.0 \mathrm{~mL}$ of $2.5 \mathrm{M})$ at $43{ }^{\circ} \mathrm{C}$ under atmospheric conditions. The isolated cellulose samples were then collected by filtration and rinsed with an excess of DI filtered water, and dried in the fume hood. This procedure is described as hemicellulose removal by acid hydrolysis or the resulting samples as hemi-removed (Hemi) thoughout this paper. The cellulose after isolation was never dried and after their initial analysis stored in a freezer at $-4.0{ }^{\circ} \mathrm{C}$ to maintain a moisture content greater than $30 \%$. 


\subsection{NMR Analysis}

The NMR samples were prepared with moist isolated cellulose (60-30\% water content) packed into 4-mm cylindrical ceramic MAS rotors. Solid-state NMR measurements were carried out on a Bruker DSX-400 spectrometer operating at frequencies of $43.55 \mathrm{MHz}$ for ${ }^{13} \mathrm{C}$ in a Bruker double-resonance MAS probehead at spinning speeds of $10 \mathrm{kHz}$. CP/MAS experiments utilized a $5 \mu \mathrm{s}\left(90^{\circ}\right)$ proton pulse, $1.5 \mathrm{~ms}$ contact pulse, $4 \mathrm{~s}$ recycle delay and 4-8 $\mathrm{K}$ scans. Line-fitting analyses of the spectra were performed using NUTS NMR Data Processing software (Acorn NMR, Inc., Livermore, CA, USA) [58]. A representative statistical average and standard deviation for the various \% crystallinity values was generated from isolated cellulose over 5 different trials from untreated Populus, which was measured for $\%$ crystallinity values by NMR after extraction, holocellulose pulping and $\mathrm{HCl}$ treatment. The standard deviation for the \% crystallinity values was $\sim 0.6 \%$, and therefore we report a typical error of $\sim \pm 2 \%$. A similar error analysis was used to determine the typical error associated with $\%$ cellulose $\mathrm{I}_{\alpha}, \mathrm{I}_{\beta}$ and para-crystalline cellulose along with cellulose fibril dimensions in Table 1 .

\section{Conclusions}

The removal of lignin by holocellulose pulping and hemicellulose removal by acid hydrolysis was shown to have little effect on the crystalline ultrastructural components of cellulose based on NMR and GPC results of microcrystalline cellulose. The results of the same experiments on cotton suggest there are some significant effects on amorphous cellulose forming spatially localized acid hydrolysis during lignin and hemicellulose removal. This spatially localized degradation manifests as a change in both cellulose DP and crystallinity. However, based on previous data collected by Hubbell et al. [53] and the time-resolved study of the hemicellulose removal on holocellulose pulped Populus, these effects seem to be mitigated by the presence of lignin and hemicellulose.

The results of this study also suggest the traditional method of acid catalyzed removal of hemicelluloses is the most effective, though the corresponding degradation in cellulose DP can be significant as seen in Figures 5 and 6 . The data suggests this degradation is invariably accompanied by changes in the crystallinity of cellulose as seen in Figure 4. Although, when limited to nominal cellulosic degradation, approximately less than two cellulose chain breaks, the increase in CrI was consistently $\sim 10 \%$ or less. In some cases where the biomass was uncharacteristically low in hemicellulose or crystalline cellulose contents, empirically reducing the acid hydrolysis times can be very important in obtaining accurate degree of crystallinity and ultrastructural information. However, based on the conditions studied for Populus pulps, MCC and cotton in this report, accurate NMR cellulose spectra, values of cellulose crystallinity and crystalline allomorphs can be ascertained when holocellulose pulps are treated with $2.5 \mathrm{M} \mathrm{HCl}$ at $43{ }^{\circ} \mathrm{C}$ for residence times between 1.5 and $4 \mathrm{~h}$.

\section{Acknowledgements}

This research was funded by the Genomic Science Program, Office of Biological and Environmental Research, U.S. Department of Energy, under FWP ERKP752. In addition, we wish to thank the reviewers for their insightful comments. 


\section{References and Notes}

1. Pu, Y.; Zhang, D.; Singh, P.M.; Ragauskas A.J. The new forestry biofuels sector. Biofuels Bioprod. Bioref. 2008, 2, 58-73.

2. Ragauskas, A.J.; Williams, C.K.; Davison, B.H.; Britovsek, G.; Cairney, J.; Eckert, C.A.; Frederick, W.J., Jr.; Hallett, J.P.; Leak, D.J.; Liotta, C.L.; et al. The path forward for biofuels and biomaterials. Science 2006, 311, 474-479.

3. Himmel, M.E.; Ding, S.-Y.; Johnson, D.K.; Adney, W.S.; Nimlos, M.R.; Brady, J.W.; Foust, T.D. Biomass recalcitrance: Engineering plants and enzymes for biofuels production. Science 2007, 315, 804-807.

4. Hsu, T.A.; Ladisch, M.R.; Tsao, T. Alcohol from cellulose. Chem. Technol. 1980, 10, 315-319.

5. Lee, S.B.; Kim, I.H.; Ryu, D.D.; Taguchi, H. Structural properties of cellulose and cellulase reaction mechanism. Biotechnol. Bioeng. 1983, 25, 33-51.

6. Foston, M.; Ragauskas, A.J. Changes in lignocellulosic supramolecular and ultrastructure during dilute acid pretreatment of Populus and switchgrass. Biomass Bioenergy 2010, 34, 1885-1898.

7. Foston, M.; Ragauskas, A.J. Changes in the structure of the cellulose fiber wall during dilute acid pretreatment in Populus studied by ${ }^{1} \mathrm{H}$ and ${ }^{2} \mathrm{H}$ NMR. Energy Fuels 2010, 24, 5677-5685.

8. Joeh, T.; Ishizawa, C.I.; Davis, M.F.; Himmel, M.E.; Adney, W.S.; Johnson, D.K. Cellulase digestibility of pretreated biomass is limited by cellulose accessibility. Biotechnol. Bioeng. 2007, 98, 112-122.

9. Thompson, D.N.; Chen, H.C.; Grethlein, H.E. Comparison of pretreatment methods on the basis of available surface area. Bioresour. Technol. 1992, 39, 155-163.

10. Kumar, P.; Barrett, D.M.; Delwiche, M.J.; Stroeve, P. Methods for pretreatment of lignocellulosic biomass for efficient hydrolysis and biofuel production. Ind. Eng. Chem. Res. 2009, 48, 3713-3729.

11. Mosier, N.; Wyman, C.; Dale, B.; Elander, R.; Lee, Y.Y.; Holtzapple, M.; Ladisch, M. Features of promising technologies for pretreatment of lignocellulosic biomass. Bioresour. Technol. 2005, 96, 673-686.

12. Sun, Y.; Cheng, J. Hydrolysis of lignocellulosic materials for ethanol production: A review. Bioresour. Technol. 2002, 83, 1-11.

13. Cohen, R.; Jensen, K.A., Jr.; Houtman, C.J.; Hammel, K.E. Significant levels of extracellular reactive oxygen species produced by brown rot basidomycetes on cellulose. FEBS Lett. 2002, 531, 473-478.

14. Sinitsyn, A.P.; Gusakov, A.V.; Vlasenko, E. Effect of structural and physico-chemical features of cellulosic substrates on the efficiency of enzymatic hydrolysis. Appl. Biochem. Biotechnol. 1991, 30, 44-59.

15. Pu, Y.; Ziemer, C.; Ragauskas, A.J. CP/MAS ${ }^{13} \mathrm{C}$ NMR analysis of cellulase treated bleached softwood kraft pulp. Carbohydr. Res. 2006, 337, 591-597.

16. Zhang, Y.H.; Lynd, L.R. Toward an aggregated understanding of enzymatic hydrolysis of cellulose: Noncomplexed cellulase systems. Biotechnol. Bioeng. 2004, 88, 797-824.

17. O'Sullivan, A.C. Cellulose: The structure slowly unravels. Cellulose 1997, 4, 173-207.

18. Atalla, R.H. Cellulose. In Comprehensive Natural Products Chemistry, Barton, D.N.K., Meth-Cohn, O., Eds.; Elsevier: Amsterdam, The Netherlands, 1999; Volume 3. 
19. VanderHart, D.L.; Atalla, R.H. ${ }^{13}$ C NMR spectra of cellulose polymorphs. J. Am. Chem. Soc. 1980, 109, 3249-3250.

20. VanderHart, D.L.; Atalla, R.H. Observations by high-resolution carbon-13 nuclear magnetic resonance of cellulose I related to morphology and crystal structure. Macromolecules 1981, 14, 570-574.

21. Larsson, P.T.; Westermark, U.; Iversen, T. Determination of the cellulose I $\alpha$ allomorph content in a tunicate cellulose by CP/MAS ${ }^{13} \mathrm{C}$ NMR spectroscopy. Carbohydr. Res. 1995, 278, 339-344.

22. Larsson, P.T.; Wickholm, K.; Iversen, T. A CP/MAS ${ }^{13} \mathrm{C}$ NMR investigation of molecular ordering in celluloses. Carbohydr. Res. 1997, 302, 19-25.

23. Larsson, P.T.; Hult, E.L.; Wickholm, K.; Pettersson, E.; Iversen, T. CP/MAS ${ }^{13}$ C-NMR spectroscopy applied to structure and interaction studies on cellulose I. Solid State Nucl. Magn. Reson. 1999, 15, 31-40.

24. Wickholm, K.; Larsson, P.T.; Iversen, T. Assignment of non-crystalline forms in cellulose I by CP/MAS ${ }^{13}$ C NMR spectroscopy. Carbohydr. Res. 1998, 312, 123-129.

25. Wickholm, K.; Hult, E.L.; Larsson, P.T.; Iversen, T.; Lennholm, H. Quantification of cellulose forms in complex cellulose materials: A chemometric model. Cellulose 2001, 8, 139-147.

26. VanderHart, D.L.; Atalla, R.H. Studies of microstructure in native celluloses using solid-state carbon-13 NMR. Macromolecules 1984, 17, 1455-1462.

27. VanderHart, D.L.; Atalla, R.H. A composite of two distinct crystalline forms. Science 1984, 223, 283-285.

28. Newman, R.H. Estimation of the lateral dimensions of cellulose crystallites using ${ }^{13} \mathrm{C}$ NMR signal strengths. Solid State Nucl. Magn. Reson. 1999, 15, 21-29.

29. Newman, R.H.; Hemmingson, J.A. Carbon-13 NMR distinction between categories of molecular order and disorder in cellulose. Cellulose 1994, 2, 95-110.

30. Maunu, S.L.; Littia, T.; Kauliomaki, S.; Hortling, B.; Sundquist, J. ${ }^{13}$ C CPMAS NMR investigations of cellulose polymorphs in different pulps. Cellulose 2000, 7, 146-159.

31. Lennholm, H.; Larsson, P.T.; Iversen T. Determination of cellulose $I_{\alpha}$ and $I_{\beta}$ in lignocellulosic materials. Carbohydr. Res. 1994, 261, 119-131.

32. Liitiä, T. Application of Modern NMR Spectroscopic Techniques to Structural Studies of Wood and Pulp Components; University of Helsinki: Helsinki, Finland, 2002.

33. Huex, L.; Dinand, E.; Vignon, M.R. Structural aspects in ultrathin cellulose microfibrils followed by ${ }^{13}$ C CP-MAS NMR. Carbohydr. Polym. 1999, 40, 115-124.

34. Hult, E.L.; Larsson, P.T.; Iversen, T. A comparative CP/MAS ${ }^{13}$ C-NMR study of cellulose structure in spruce wood and kraft pulp. Cellulose 2000, 7, 35-55.

35. Hult, E.L.; Larsson, P.T.; Iversen, T. Cellulose fibril aggregation-An inherent property of kraft pulps. Polymer 2001, 42, 3309-3314.

36. Hult, E.L.; Larsson, P.T.; Iversen, T. A comparative CP/MAS ${ }^{13} \mathrm{C}-\mathrm{NMR}$ study of the supermolecular structure of polysaccharides in sulphite and kraft pulps. Holzforschung 2002, 56, 179-184.

37. Hult; E.L.; Liitia, T.; Maunu, S.L.; Hortling, B.; Iversen, T. A CP/MAS 13C-NMR study of cellulose structure on the surface of refined kraft pulp fibers. Carbohydr. Polym. 2002, 49, 231-234. 
38. Hult, E.L.; Iversen, T.; Sugiyama, J. Characterization of the supermolecular structure of cellulose in wood pulp fibres. Cellulose 2003, 10, 103-110.

39. Newman, R.H.; Hemmingson, J.A. Determination of the degree of cellulose crystallinity in wood by Carbon-13 nuclear magnetic resonance spectroscopy. Holzforschung 1990, 44, 351-355.

40. Leary, G.; Morgan, K.; Newman, R.H. A ${ }^{13}$ C CP/MAS NMR comparison of wood fractions from spruce. Holzforschung 1986, 40, 221-224.

41. Park, S.; Johnson, D.K.; Ishizawa, C.; Parilla, P.; Davis, M. Measuring the crystallinity index of cellulose by solid state ${ }^{13} \mathrm{C}$ nuclear magnetic resonance. Cellulose 2009, 16, 641-646.

42. Ding, S.; Himmel, M. The maize primary cell wall microfibril: A new model derived from direct visualization. J. Agric. Food Chem. 2006, 54, 597-606.

43. Tang, H.; Wang, Y. High resolution solid-state NMR spectroscopy of starch polysaccharides. Mod. Magn. Reson. 2006, 3, 1761-1769.

44. Rowland, S.P.; Roberts, E.J.; Bose, J.L.; Wade, C.P. Assessment of the state of order of hydroxyl groups and of molecular segments in hydrocellulose. J. Polym. Sci. Part A 1971, 9, 1623-1633.

45. Kolpak, F.; Weih, M.; Blackwell, J. Assessment of the state of order of hydroxyl groups and of molecular segments in hydrocellulose. Polymer 1978, 19, 123-131.

46. Langan, P.; Nishiyama, Y.; Chanzy, H. X-ray structure of mercerized cellulose II at $1 \AA$ resolution. Biomacromolecules 2001, 2, 410-416.

47. Park, S.; Baker, J.O.; Himmel, M.; Parilla, P.A.; Johnson, D.K. Cellulose crystallinity index: Measurement techniques and their impact on interpreting cellulase performance. Biotechnol. Biofuels 2010, 3, 10:1-10:10.

48. Heux, L.; Dinand, E.; Vignon, M.R. Structural aspects in ultra-thin cellulose microfibrils followed by ${ }^{13}$ C CP-MAS NMR. Carbohydr. Polym. 1999, 40, 115-124.

49. Newman, R.H. Evidence of assignment of ${ }^{13} \mathrm{C}$ NMR signals to cellulose crystallite surfaces in wood, pulp and isolated cellulose. Holzforschung 1998, 52, 157-159.

50. Teleman, A.; Larsson, P.T.; Iversen, T. On the accessibility and structure of xylan in birch kraft pulp. Cellulose 2001, 8, 209-215.

51. Hemmingson, J.A.; Newnan, R. A CP/MAS ${ }^{13} \mathrm{C}$ NMR study of the effect of steam explosion processes on wood composition and structure. J. Wood Chem. Technol. 1985, 5, 159-188.

52. Zhang, Y.; Lynd, L. Determination of the number-average degree of polymerization of cellodextrins and cellulose with application to enzymatic hydrolysis. Biomacromolecules 2005, 6 , 1510-1515.

53. Hubbell, C.; Ragauskas, A.J. Effect of acid-chlorite delignification on cellulose degree of polymerization. Bioresour. Technol. 2010, 101, 7370-7375.

54. Wood, B.F.; Conner, A.H.; Hill, C.G., Jr. The effect of precipitation on the molecular weight distribution of cellulose tricarbanilate. J. Appl. Polym. Sci. 1986, 32, 3703-3712.

55. Stephens, C.; Whitmore, P.; Morris, H.; Bier, M. Hydrolysis of the amorphous cellulose in cotton-based paper. Biomacromolecules 2008, 9, 1093-1099.

56. Holtman, K. An Investigation of the Milled Wood Lignin Isolation Procedure by Solution- and Solid-State NMR Spectroscopy. Ph.D. Dissertation, North Carolina State University, Raleigh, NC, USA, 2003. 
57. Davis, M. A rapid modified method for compositional carbohydrate analysis of lignocellulosics by high $\mathrm{pH}$ anion-exchange chomatography with pulsed amperometric detection (HPAEC/PAD). J. Wood Chem. Technol. 1998, 18, 235-252.

58. NutsPro-NMR Utility Transform Software-Professional; Acorn NMR: Livermore, CA, USA, 1993. Available online: http://www.acornnmr.com (accessed on 30 September 2011).

(C) 2011 by the authors; licensee MDPI, Basel, Switzerland. This article is an open access article distributed under the terms and conditions of the Creative Commons Attribution license (http://creativecommons.org/licenses/by/3.0/). 